\title{
Memory for Temporal Order of New and Familiar Spatial Location Sequences: Role of the Medial Prefrontal Cortex
}

\author{
Andrea A. Chiba, Raymond P. Kesner, ${ }^{1}$ and Chris J. Gibson \\ Department of Psychology \\ University of Utah \\ Salt Lake City, Utah 84112
}

\begin{abstract}
Rats with medial prefrontal cortex or sham control lesions were tested on an eight-arm radial maze task to examine memory for the temporal order of a variable and a constant sequence of spatial locations as a function of temporal distance. During the study phase of each trial, rats were allowed to visit each of eight arms once in an order that was randomly selected or fixed for that trial. The test phase required the rats to choose which of two arms occurred earlier in the sequence of arms visited during the study phase. The arms selected as test arms varied according to temporal distance $(0,2,4$, or 6$)$ or the number of arms that occurred between the two test arms in the study phase.

For the variable sequences based on new information, control rats showed an increasing temporal distance function. Relative to control rats, medial prefrontal cortex-lesioned rats displayed a temporal order memory deficit across all distances. For the constant sequence based on familiar information, control rats performed well across all distances. Relative to controls, the medial prefrontal cortex-lesioned rats displayed a performance deficit. The results support the idea that the medial prefrontal cortex contributes to mnemonic operations associated with temporal order for new and familiar spatial location information.
\end{abstract}

\section{Introduction}

There are at least three major components into

${ }^{1}$ Corresponding author. which memory for time can be divided (Block 1990). First, there is succession, temporal order, or relative recency, referring to memory for the sequential occurrence of events. Second, there is memory for duration of events as well as memory for intervals or time periods between events. Third, there is memory for time perspective based on memory for the past, present, and future. Rats have been shown to be rather accurate in judgments of elapsed time or duration. This accuracy extends to memory for the duration of an event, reflecting the operation of a memory system for time as duration (Church and Gibbon 1982; Olton 1986; P. Jackson-Smith, R.P. Kesner, and K. Amann, unpubl.). Rats have also been shown to remember accurately the order of presentation of a series of specific events (spatial locations) and the frequency of occurrence of specific events (spatial locations), reflecting memory for succession or temporal order (Kesner et al. 1984; Kesner $1990 a, b)$. Finally, rats have been shown to remember the past and the future based on the utilization of prospective and retrospective strategies (Cook et al. 1985; Kametani and Kesner 1989).

Previous research has indicated that the medial prefrontal cortex (MPF) in the rat or human does not disrupt memory for event duration (P. JacksonSmith, R.P. Kesner, K. Amann, unpubl.; R.O. Hopkins and R.P. Kesner, unpubl.) but does disrupt memory for future events based on the ability to use a prospective code (Shallice 1982; Kesner 1989). Other research has shown that MPF lesions in the rat disrupt memory for the temporal order of a list of spatial locations as well as memory for the frequency of occurrence of specific spatial locations within a list (Kesner and Novak 1982; Kesner and Holbrook 1987; Kesner 1990a,b). Similar order or relative recency memory deficits have been observed in human patients or monkeys with dorsolateral frontal cortex lesions (Milner 1971; Shi-

LEARNING \& MEMORY 4:311-317 @ 1997 by Cold Spring Harbor Laboratory Press ISSN1072-0502/97 \$5.00

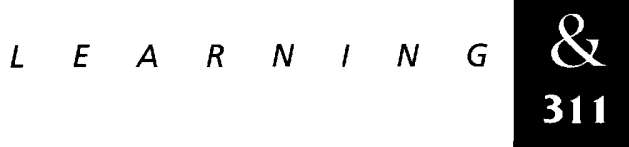

$\begin{array}{llllll}M & E & M & O & R & Y\end{array}$ 
Chiba et al.

mamura et al. 1990; Petrides 1991; Kesner et al. 1994). These observations are consistent with the suggestion that the MPF is a critical substrate for coding the temporal order of newly acquired or extant information.

In a recent study it was shown that rats display a stable temporal distance function (i.e., improved memory performance as the number of items between test items presented in the study phase exceeds zero) for spatial location information (Chiba et al. 1994). This distance function is similar to what has been obtained in humans for a variety of stimuli including words and spatial locations (Kesner et al. 1992; Madsen and Kesner 1995). Furthermore, it was shown in the Chiba et al. (1994) study that lesions of the MPF produced profound deficits in the temporal distance function. It was proposed that the MPF codes the occurrence of each event across time, so that a chronological order of experiences can be retrieved at some later time, and, in addition, it was assumed that the MPF mediates temporal organizational strategies and rules so that the information remains organized in an appropriate sequence. To test this latter idea more directly, the temporal distance paradigm was extended to include not only variable study phase sequences requiring order memory for new sequences but also one constant study phase sequence requiring order memory for a well-learned sequence. It is predicted that MPF lesions would display a deficit for the variable sequences as well as the welllearned constant sequence.

\section{Materials and Methods}

\section{SUBJECTS}

Fourteen male Long-Evans hooded rats weighing between 300 grams and 400 grams were used as subjects. Each rats was individually housed and maintained at $\sim \mathbf{8 5} \%-\mathbf{9 0 \%}$ of its free-feeding weight. The rat cages were kept in a laboratory with a 12-hr light-dark cycle.

\section{APPARATUS}

An eight-arm radial maze served as the test apparatus. The maze was painted white, stood 100 $\mathrm{cm}$ above the floor, and was kept in a well-lit room having no windows, one door, and four calendar pictures that were placed on the walls of the room.
The maze consisted of an octagonal central platform with a diameter of $42 \mathrm{~cm}$. Eight arms radiated from the central platform like spokes of a wheel. The arms were $71 \mathrm{~cm}$ long $\times 9.5 \mathrm{~cm}$ wide and were attached to the central platform with metal braces.

Each arm had 0.3 -cm-thick clear Plexiglas sides, which rose $5.7 \mathrm{~cm}$ above the surface of the arm and extended from the distal end of the arm to $2.5 \mathrm{~cm}$ from the central platform. A food well, 2.5 $\mathrm{cm}$ in diameter, was drilled $1.5 \mathrm{~cm}$ deep at the distal end of each arm. A $0.3-\mathrm{cm}$ Plexiglas guillotine door was located at the juncture between the platform and the arm. Each door was $10 \mathrm{~cm}$ wide and, when in the raised position, extended $18 \mathrm{~cm}$ above the surface of the platform. By means of a series of pulleys and strings, each door could be raised and lowered.

Strips of Plexiglas were installed on the central platform, in the gaps between the doors, to prohibit the rats from climbing between the doors. These strips, together with the doors, effectively formed a cylindrical Plexiglas chamber on the central platform into which the rats could be placed through the open top. Finally, a linoleum cylinder, $32 \times 122 \mathrm{~cm}$, could be placed inside the central chamber allowing the rat to see only the ceiling of the lab.

\section{PRETRAINING}

Each rat was adapted to the eight-arm radial maze for 14 days. For the first 7 days, each rat received one training trial per day during which the rat was placed on the central platform of the maze with all of the Plexiglas doors in the open position. At this time, food (Froot Loops cereal) was available in the center of the maze and at the end of the arms. The rat was allowed to explore the maze and eat until either the food source was depleted or $10 \mathrm{~min}$ had elapsed. During days 7 through 14 of the training period, a similar procedure was followed with the exception that food was available only in the food wells at the end of the arms. Additionally, at the beginning of each trial, four of the doors were left in the open position, whereas the remaining four doors were in the closed position. After the rat depleted the food accessed through the four open doors, the experimenter opened each of the closed doors individually. Following the familiarization training, the animals commenced the temporal order task. Animals

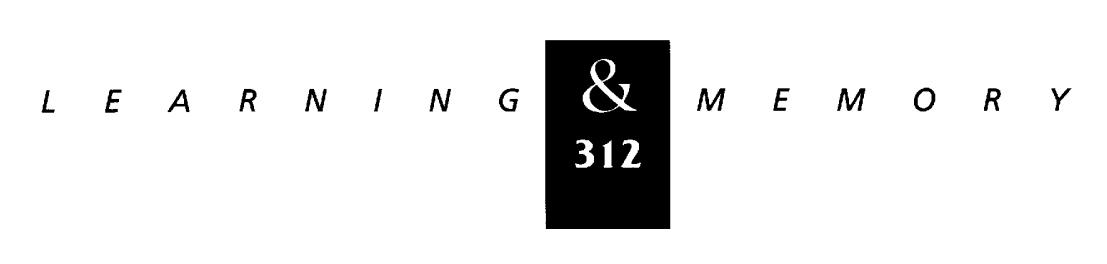


received one trial a day. Each trial consisted of a study phase and a test phase. During the study phase of half of the trials, the rat was allowed to visit each of eight arms once in an order that was randomly selected for that trial (variable sequence). During the other half of the trials, the rat was given a sequence that did not vary from trial to trial (constant sequence). The variable and constant sequence trials alternated on a daily basis. The sequence of the eight arms was presented to the rat by the sequential opening of Plexiglas doors (one at a time) located at the entrance of each arm. Immediately after the animal received reinforcement from the last of the eight arms (e.g., completed the study phase), the test phase was begun. Only one test was given for each trial and consisted of opening two doors simultaneously. Temporal distances of $0,2,4$, or 6 were randomly selected for each test phase and were represented as the number of maze arms that occurred between the two test arms in the study phase. For the test of a temporal distance of 0 , the arms followed each other in the study phase. For the test of a temporal distance of 6 , six arms occurred between the two test arms in the study phase. The rule to be learned to obtain a reinforcement in the test phase was to choose the arm that occurred earlier in the sequence. Rats were trained until they reached a criterion of $75 \%$ correct for each temporal distance, with the exception of the 0 distance for both the variable and constant conditions, based on 64 trials with eight tests randomly selected for each temporal distance condition. If this criterion was met, surgery was performed.

\section{SURGERY}

In preparation for surgery, rats were anesthetized with Nembutal (50 $\mathrm{mg} / \mathrm{kg}$, i.p.) and were given atropine sulfate $(0.2 \mathrm{mg} / \mathrm{kg}, \mathrm{i} . \mathrm{p}$. $)$ as a prophylactic. Rats in all groups were given a midline incision that was sutured following surgery. The first group of rats $(n=9)$ received bilateral aspiration lesions of the MPF. The lesions extended from bregma anterior to the frontal pole and included an area $1.5 \mathrm{~mm}$ on each side of midline and $3 \mathrm{~mm}$ below dura. The last group of rats $(n=5)$ served as sham-operated controls. Beginning 1 week following surgery, all animals were given 64 trials with 8 trials at each temporal distance including variable and constant conditions.

\section{HISTOLOGY}

At the end of the experiment, animals were given a lethal intraperitoneal injection of sodium pentobarbital. The rats were then perfused with $10 \%$ buffered formalin in $0.1 \mathrm{M}$ phosphate buffer. The brain was removed and embedded in a bovine serum albumin matrix. Serial sections (30 $\mu \mathrm{m})$ were processed using a freezing microtome, mounted in a 1 in 5 series on gelatin-covered slides, and stained with cresyl violet.

\section{Results}

HISTOLOGY

A schematic representation of the smallest and largest MPF lesions across four anterior-posterior levels is shown in Figure 1. The small and large MPF-lesioned brains included damage to the medial precentral, dorsal-medial, and ventral anterior cingulate subregions of the MPF. There was very little damage to ventrolateral anterior cingulate or prelimbic and infralimbic cortex. There was little damage to the dorsolateral cortex for the smallest lesion but more extensive damage to the dorsolateral cortex for the largest lesion (Groenewegen 1988).

\section{BEHAVIORAL ANALYSIS}

In Figures 2, $\mathrm{A}$ and $\mathrm{B}$, and 3, A and B, pre- and postsurgical percent correct performance is shown as a function of temporal distance for each lesion condition. Figure 2 , A and B, indicates that performance of control rats did not vary as a result of surgery and that there was a temporal distance effect in the variable condition. Figure 3, A and B, indicates that MPF lesions produced a profound deficit in both the variable and constant conditions. The effects appear to be greater for a distance of 4 in both conditions.

An overall fourway analysis of variance (ANOVA) with lesion groups as the between factor and pre-post, constant versus variable, and distance as within factors revealed a significant lesion effect $(F=95.7, d f=1,12, P<0.0001)$, a significant pre-post effect $(F=25.5, d f=1,12, P<$ 0.0003 ), a significant constant versus variable effect $(F=7.99, d f=1,12, P<0.015)$, a significant distance effect $(F=13.3, d f=3,36, P<0.0001)$, a significant lesion by pre-post interaction $(F=34.5$, $d f=1,12, P<0.001)$, and a significant triple inter-

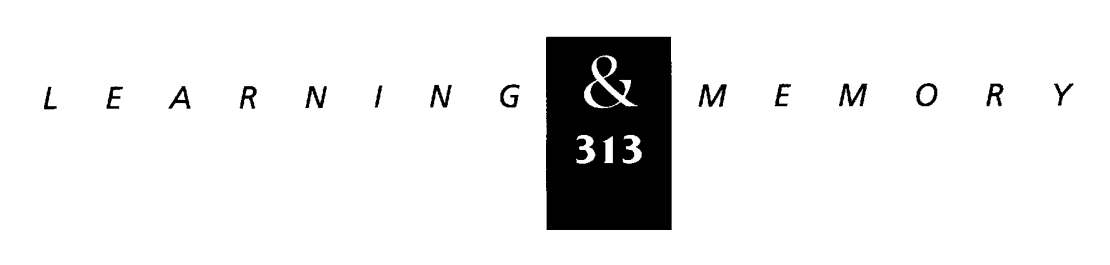



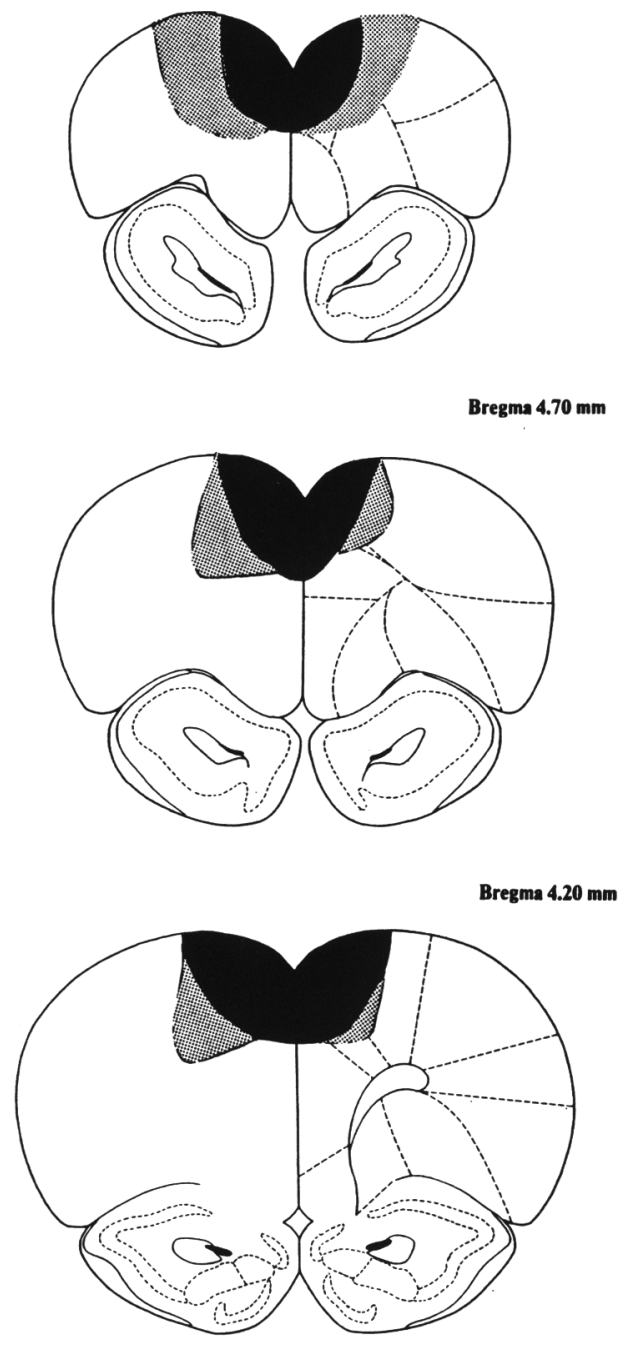

Bregma $3.70 \mathrm{~mm}$

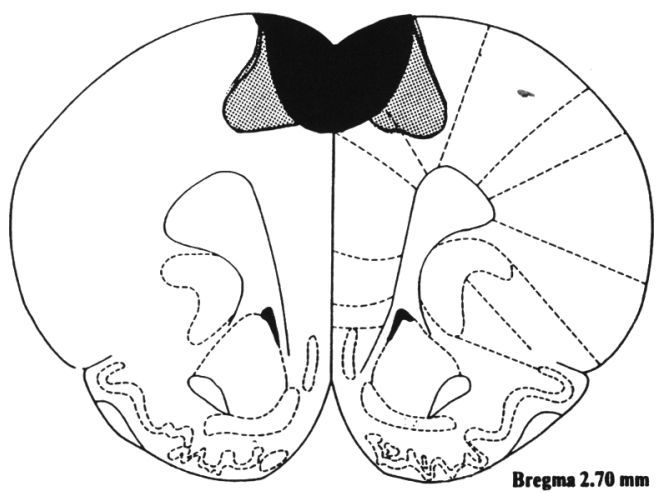

Figure 1: A schematic representation of the smallest and largest medial prefrontal cortex lesions across four anterior-posterior levels.

action among pre-post, constant versus variable, and lesion group $(F=6.7, d f=1,12, P<0.023)$. A
Newman-Keuls test on the distance effect revealed that performance for the distance of 6 was significantly higher than performance for the distance of $0(P<0.05)$. A Newman-Keuls test was also used to analyze the triple interaction effect. The test revealed that the groups did not differ on the presurgery tests for the constant or variable conditions. However, on the postsurgery tests for both the variable and the constant conditions, the MPF group was significantly different from control $(P<0.05)$.

Based on a separate analysis on only the con-

A

CONTROL
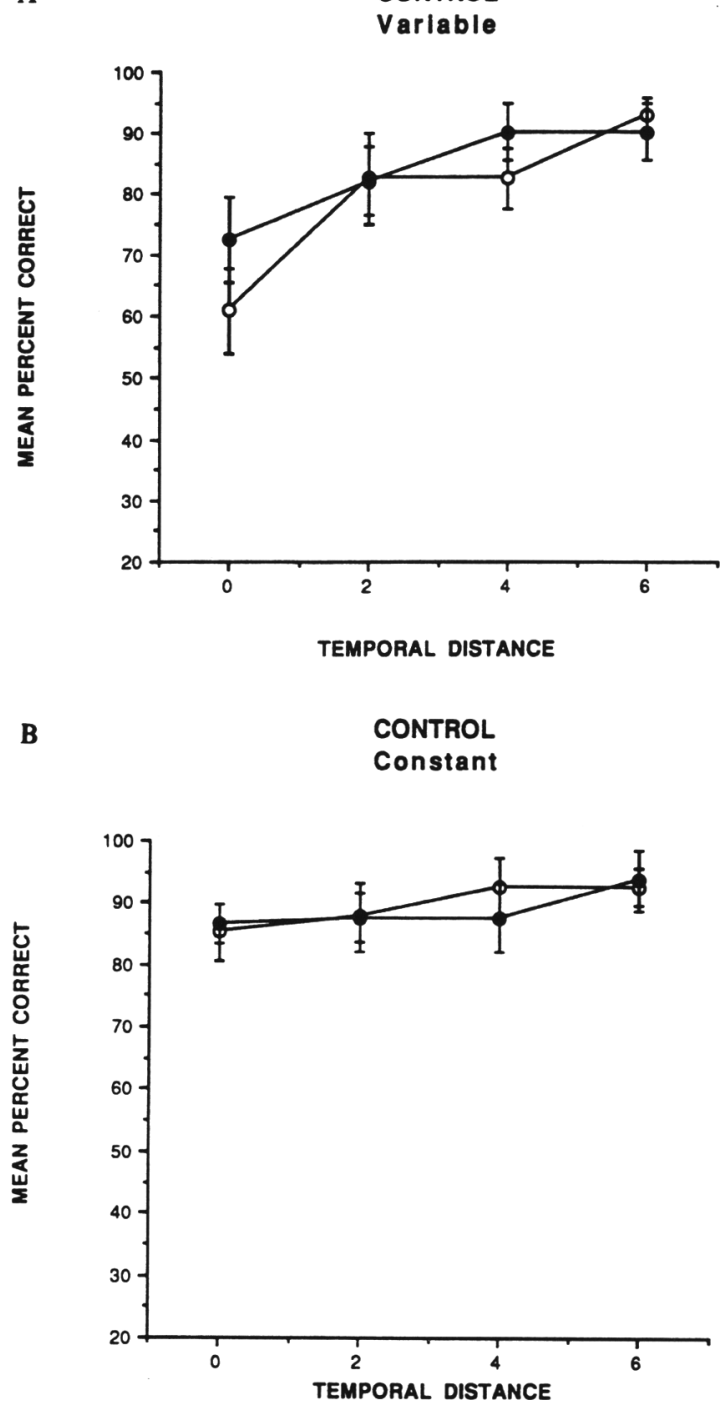

Figure 2: Mean percent correct performance \pm S.E.) for presurgical and postsurgical control animals as a function of temporal distance for variable sequences $(A)$ and a constant sequence $(B)$. 
$\mathbf{A}$
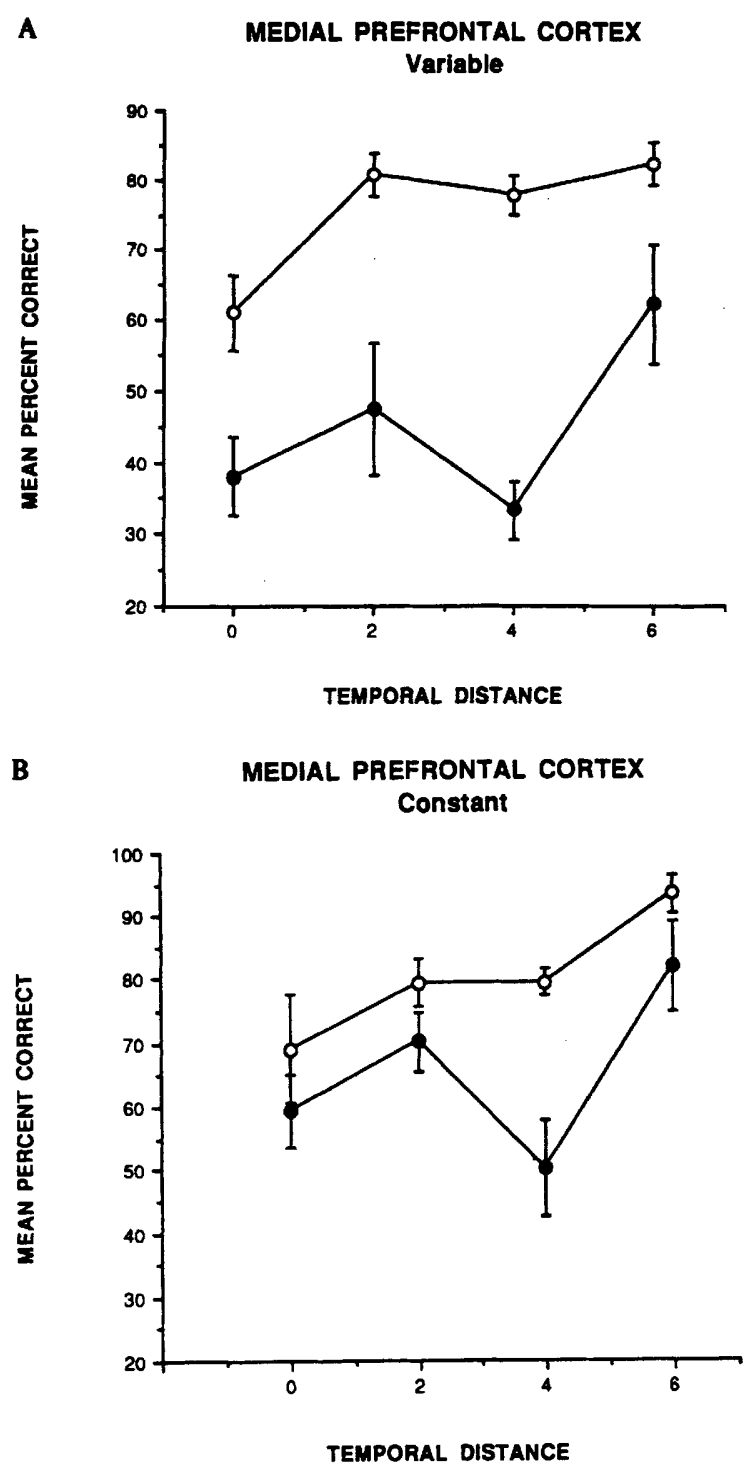

Figure 3: Mean percent correct performance ( \pm S.E.) for presurgical and postsurgical medial prefrontal cortexlesioned animals as a function of temporal distance for variable sequences $(A)$ and a constant sequence $(B)$.

trol group, a Newman-Keuls test across distances revealed that there was a significant reduction in performance for the 0 distance in comparison with distances of 2,4 , or $6(P<0.05)$ in the variable condition but no significant differences among the distances in the constant condition.

Because of a concern that performance on the 4 distance position of the MPF group might be the major contributor to the deficits seen in the constant and variable postsurgery conditions, a separate analysis on only the postsurgery tests for the MPF group was performed. A Newman-Keuls test across distances revealed that for both the constant and variable conditions, the performances for the 4 and 0 distance positions were significantly different from the 6 distance position $(P<0.05)$. Furthermore, performance on the 4 distance position did not differ from the 0 or 2 distance positions. Thus, the observed deficits in the constant and variable postsurgery conditions for the MPF-lesioned rats are not primarily attributable to the performance on the distance 4 position.

\section{Discussion}

The results of the present experiment indicate that the performance of control rats in the variable condition resulted in a temporal distance function, which appeared to be stable over time. In examining the temporal distance function displayed by control rats, it is evident that even though a monotonic relationship between mean percent correct performance and temporal distance exists, the critical distinction lies between performance on the temporal distance of 0 and performance on those distances $>0$. This form of the distance function in the variable condition replicates our previous study (Chiba et al. 1994), even though the performance at the 0 distance was higher in the present study. In the previous study (Chiba et al. 1994), it was suggested that the improved memory performance with increasing distance was owing to the possibility that at the time of presentation, the rat encodes a sample of the internal or external context as a crucial component of the mnemonic trace of each spatial location. Thus, variation of context serves as a mnemonic aid in making order discriminations. In the instance of the temporal distance 0 , the context of the test items does not differ sufficiently to aid in making an accurate order discrimination. Support for this explanation can be found in studies of human memory (Madigan 1969; Bennett 1975) in which a disproportionate effect on recall or discrimination was exerted by the utilization of a distance of 0 or 1 as compared with distance $>0$ or 1 . Thus, the idea of discriminability of internal or external context can readily account for the observed temporal distance function in the variable condition. The results also indicate an absence of a temporal distance function in the constant condition, which could be attributable to the reduction or elimination of contextual variations in the constant condition.

The results of the present experiment also in-

$$
\begin{array}{llllllllllllllll}
\hline & E & A & R & N & I & N & G & \mathbf{Q} & M & E & M & O & R & Y
\end{array}
$$


dicate that compared to presurgery performance, rats with MPF lesions are impaired in temporal order memory for spatial locations in both the variable and constant conditions. These data are consistent with a previous study in which MPF lesions were affected in the variable condition (Chiba et al. 1994). These data are also consistent with previous findings demonstrating that MPF lesions disrupt temporal order memory for all items within an eight-item spatial location list as well as memory for the frequency of spatial locations (Kesner and Holbrook 1987; Kesner 1990a; Chiba et al. 1994). Similar temporal order and frequency deficits to those observed in rats have been observed in human patients with frontal lobe damage (Milner 1971; Smith and Milner 1988; Kesner and JacksonSmith 1992; Kesner et al. 1994). Humans with frontal lobe damage are not impaired in recognition memory for specific items (spatial location, words, or abstract pictures), but they are impaired in order recognition memory and in judgements of frequency for the same items. The order memory deficits observed in rats with MPF lesions are not likely to be attributable to their inability to remember spatial locations. This is supported by the observation that rats with MPF lesions are not impaired when recognition tests are used for a single spatial location or a single visual object (Milner 1971; Kesner and Jackson-Smith 1992; R.P. Kesner, unpubl.) and by the observation that for a constant sequence, rats with MPF lesions are impaired in remembering the order of presentation but have no difficulty in remembering the items (spatial location) that were presented (Kesner and Holbrook 1987). These results are consistent with the suggestion that the MPF mediates event classification rules (Wise et al. 1996), which can be used in remembering well-organized temporal sequences.

In general, the data suggest that the MPF contributes to mnemonic operations associated with temporal order memory for spatial location information. It appears that the MPF is critical in separating the occurrence of events across time, so that a chronological order of experiences can be retrieved at some later time. In addition, the MPF organizes repeated sequential occurrences of events in long-term memory so that temporal classification rules can be used for efficient retrieval of meaningful temporal sequences.

\section{Acknowledgments}

This research was supported by National Institutes of
Health grant 2R01NS20771-13 and National Science Foundation grant BNS 892-1532.

The publication costs of this article were defrayed in part by payment of page charges. This article must therefore be hereby marked "advertisement" in accordance with 18 USC section 1734 solely to indicate this fact.

\section{References}

Bennett, P.W. 1975. Proactive interference in short-term memory: Fundamental forgetting processes. J. Verbal Learn. Verbal Behav. 14: 123-144.

Block, R.A. 1990. Models of psychological time. In Cognitive models of psychological time (ed. R.A. Block), pp. 1-36. Lawrence Erlbaum, Hillsdale, NJ.

Chiba, A.A., R.P. Kesner, and A.M. Reynolds. 1994. Memory for spatial location as a function of temporal distance in rats: Role of hippocampus and medial prefrontal cortex. Behav. Neural Biol. 61: 123-131.

Church, R.M. and J. Gibbon. 1982. Temporal generalization J. Exp. Psychol. Anim. Behav. Processes 8: 165-186.

Cook, R.G., M.F. Brown, and D.A. Riley. 1985. Flexible memory processing by rats: Use of prospective and retrospective information in the radial maze. J. Exp. Psychol. Anim. Behav. Processes 11: 453-469.

Groenewegen, J.J. 1988. Organization of the afferent connections of the mediodorsal thalamic nucleus in the rat, related to the mediodorsal-prefrontal topography. Neuroscience 24: 379-431.

Kametani, H. and R.P. Kesner. 1989. Retrospective and prospective coding of information: Dissociation of parietal cortex and hippocampal formation. Behav. Neurosci. 103: 84-89.

Kesner, R.P. 1989. Retrospective and prospective coding of information: Role of the medial prefrontal cortex. J. Exp. Brain Res. 74: 163-167.

1990a. Memory for frequency in rats: Role of the hippocampus and medial prefrontal cortex. Behav. Neural Biol. 53: 402-410.

1990b. Learning and memory in rats with an emphasis on the role of the hippocampal formation. In Neurobiology of comparative cognition (ed. R.P. Kesner and D.S. Olton), pp. 179-204. Lawrence Erlbaum, Hillsdale, NJ.

Kesner, R.P. and J. Novak. 1982. Serial position curve in rats: Role of the dorsal hippocampus. Science 218: 173-174.

Kesner, R.P. and T. Holbrook. 1987. Dissociation of item and order spatial memory in rats following medial prefrontal cortex lesions. Neuropsychologia 25: 653-664.

Kesner, R.P. and P. Jackson-Smith. 1992. Neurobiology of an attribute model of memory: Role of prefrontal cortex. In Learning and memory: Behavioral and biological processes 
(ed. 1. Gormezano and E.A. Wasserman), pp. 251-273.

Lawrence Erlbaum, Hillsdale, NJ.

Kesner, R.P., M.O. Measom, S.L. Forsman, and T.H.

Holbrook. 1984. Serial position curves in rats: Order memory for episodic spatial events. Anim. Learn. Behav. 12: 378-382.

Kesner, R.P., R.O. Hopkins, and A.A. Chiba. 1992. Learning and memory in humans with an emphasis on the role of the hippocampus. In Neuropsychology of memory (ed. L. Squire and N. Butters), 2nd ed., pp. 106-121. The Guilford Press, New York, NY.

Kesner, R.P., R.O. Hopkins, and B. Fineman. 1994. Item and order dissociation in humans with prefrontal cortex damage. Neuropsychologia 32: 881-891.

Madigan, S.A. 1969. Intraserial repetition and coding precesses in free recall. J. Verbal Learn. Verbal

Behav.8: 828-835.

Madsen, J. and R.P. Kesner. 1995. The temporal distance effect in subjects with dementia of the Alzheimer's type. Alzheimer's Dis. Associated Disorders 9: 94-100.

Milner, B. 1971. Interhemispheric differences in the localization of psychological processes in man. Brain Med. Bull. 27: 272-277.

Olton, D.S. 1986. Hippocampal function and memory for temporal context. In The hippocampus (ed. R.L. Isaacson and K.H. Pribram), Vol. 4, pp. 281-298. Plenum Press, New York, NY.

Petrides, M. 1991. Functional specialization within the dorsolateral frontal cortex for serial order memory. Proc. $R$. Soc. Lond. Ser. B. 246: 299-306.

Shallice, T. 1982. Specific impairments of planning. Phil. Trans. R. Soc. Lond. B. 298: 199-209.

Shimamura, A.P., J.S. Janowsky, and L.R. Squire. 1990. Memory for the temporal order of events in patients with frontal lobe lesions and amnesic patients. Neuropsychologia 28: 803-813.

Smith, M.L. and B. Milner. 1988. Estimation of frequency of occurrence of abstract designs after frontal or temporal lobectomy. Neuropsychologia 26: 297-306.

Wise, S.P., E.A. Murray, and C.R. Gerfen. 1996. The frontal cortex-basal ganglia system in primates. Crit. Rev. Neurobiol. 10: $317-356$.

Received April 25, 1997; accepted in revised form October 30, 1997.

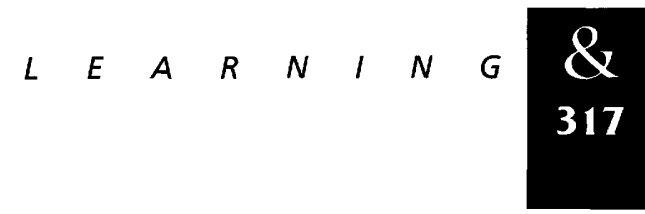




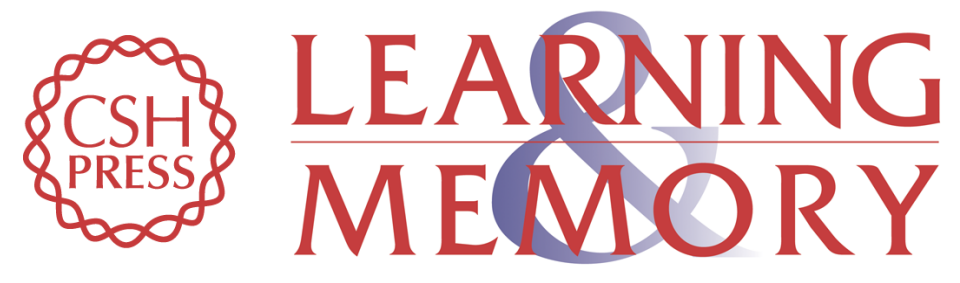

\section{Memory for temporal order of new and familiar spatial location sequences: role of the medial prefrontal cortex.}

A A Chiba, R P Kesner and C J Gibson

Learn. Mem. 1997, 4:

Access the most recent version at doi:10.1101//m.4.4.311

References This article cites 20 articles, 1 of which can be accessed free at: http://learnmem.cshlp.org/content/4/4/311.full.html\#ref-list-1

License

Email Alerting Receive free email alerts when new articles cite this article - sign up in the box at the Service top right corner of the article or click here. 